\title{
TEM10 homodyne detection as an optimal small-displacement and tilt-measurement scheme
}

Delaubert, Vincent; Treps, Nikolas; Lassen, Mikael Østergaard; Harb, Charles; Fabre, Claude; Lam, Ping Koy; Bachor, Hans A.

Published in:

Physical Review A

Link to article, DOI:

10.1103/PhysRevA.74.053823

Publication date:

2006

Document Version

Publisher's PDF, also known as Version of record

Link back to DTU Orbit

Citation (APA):

Delaubert, V., Treps, N., Lassen, M. Ø., Harb, C., Fabre, C., Lam, P. K., \& Bachor, H. A. (2006). TEM10 homodyne detection as an optimal small-displacement and tilt-measurement scheme. Physical Review A, 74(5), 053823. https://doi.org/10.1103/PhysRevA.74.053823

\section{General rights}

Copyright and moral rights for the publications made accessible in the public portal are retained by the authors and/or other copyright owners and it is a condition of accessing publications that users recognise and abide by the legal requirements associated with these rights.

- Users may download and print one copy of any publication from the public portal for the purpose of private study or research.

- You may not further distribute the material or use it for any profit-making activity or commercial gain

- You may freely distribute the URL identifying the publication in the public portal 


\title{
TEM $_{10}$ homodyne detection as an optimal small-displacement and tilt-measurement scheme
}

\author{
V. Delaubert, ${ }^{1,2}$ N. Treps, ${ }^{1}$ M. Lassen, ${ }^{2,3}$ C. C. Harb, ${ }^{2}$ C. Fabre, ${ }^{1}$ P. K. Lam, ${ }^{2}$ and H-A. Bachor ${ }^{2}$ \\ ${ }^{1}$ Laboratoire Kastler Brossel, 4 Place Jussieu, Case 74, Paris 75252 Cedex 05, France \\ ${ }^{2}$ ACQAO, The Australian National University, Canberra ACT 0200, Australia \\ ${ }^{3}$ Department of Physics, Danmarks Tekniske Universitet, Building 309, 2800 Lyngby, Denmark
}

(Received 20 July 2006; published 30 November 2006)

\begin{abstract}
We present a detailed description of small displacement and tilt measurements of a Gaussian beam using split detectors and $\mathrm{TEM}_{10}$ homodyne detectors. Theoretical analysis and an experimental demonstration of measurements of these two conjugate variables are given. A comparison between the experimental efficiency of each scheme proves that the standard split detection is only $64 \%$ efficient relative to the $\mathrm{TEM}_{10}$ homodyne detection, which is optimal for beam displacement and tilt. We also demonstrate experimentally that squeezed light in the appropriate spatial modes allows measurements beyond the quantum noise limit for both types of detectors. Finally, we explain how to choose the detection scheme best adapted to a given application.
\end{abstract}

DOI: 10.1103/PhysRevA.74.053823

PACS number(s): 42.50.Dv, 42.50.Lc

\section{INTRODUCTION}

Measuring the transverse position of a laser beam seems to be a very basic task. One could think that the best way to retrieve such a simple information has been found years ago. However, we have proven recently that the detection devices which are traditionally used - split and quadrant detectorsare limited to an efficiency of 64\% [1]. This is a question of potentially great interest as they are used in many ultrasensitive applications including optical tweezers, atomic force microscopes, beam positioning for gravitational wave detectors and satellite alignment [2-4].

The aim of this paper is to present a detailed analysis of beam displacement and tilt measurements, for a given beam power and profile, by considering two detection devices: a split detector, and a homodyne detector with an optimal local oscillator. The strength of the work presented here lies in the direct comparison of the performances of each scheme, under the same experimental conditions. Note that similar issues are addressed on the limits to the measurement of beam rotation about its propagation axis in Ref. [5]. We will first focus on the detection device, and will therefore analyze experimentally a split-detection and a homodyne-detection scheme with a TEM $_{10}$ local oscillator (see Fig. 1), introduced theoretically in Ref. [1]. At the same time, we will present measurements of the quantum conjugated variable of the transverse displacement of the beam, the laser beam tilt [6]. Finally, we will focus on the laser beam itself, and show measurements beyond the fundamental limit imposed by the photon statistics of laser beams, using nonclassical beams. This demonstration allows displacement and tilt measurements that were masked or altered by quantum noise.

The paper is organized in the following way. We first give a brief definition of displacement and tilt of a $\mathrm{TEM}_{00}$ mode beam, and introduce the notions of position and momentum of a Gaussian beam, which are two conjugate transverse observables. In Sec. III, we quantitatively discuss the QNL for displacement and tilt measurements and show the improvement that can be achieved with squeezed light. In Sec. IV, we present how this new set of quantum variables can be accessed with a split detection, the conventional scheme used for beam displacement measurements. The results obtained provide a reference for a homodyne detector with a $\mathrm{TEM}_{10}$ local oscillator presented in Sec. V. In Sec. VI, we show how to perform sub-QNL measurements with both schemes and present experimental homodyne-detection results in this regime. In Sec. VII, a comparison between both schemes is presented, in perfect agreement with theoretical predictions, and showing an improvement with the homodyne detection that matches the predicted detection efficiency of $64 \%$.

\section{DISPLACEMENT AND TILT OF A GAUSSIAN BEAM}

Displacement and tilt of a single-mode $\mathrm{TEM}_{00}$ laser beam are very intuitive notions, they refer to macroscopic properties of a beam, as shown in Fig. 2. We assume here that the beam is constrained to one dimension, namely the figure plane of the paper, considering that the nonrepresented transverse component is Gaussian. A displacement corresponds to a translation of the beam by a distance $d$ along the transverse

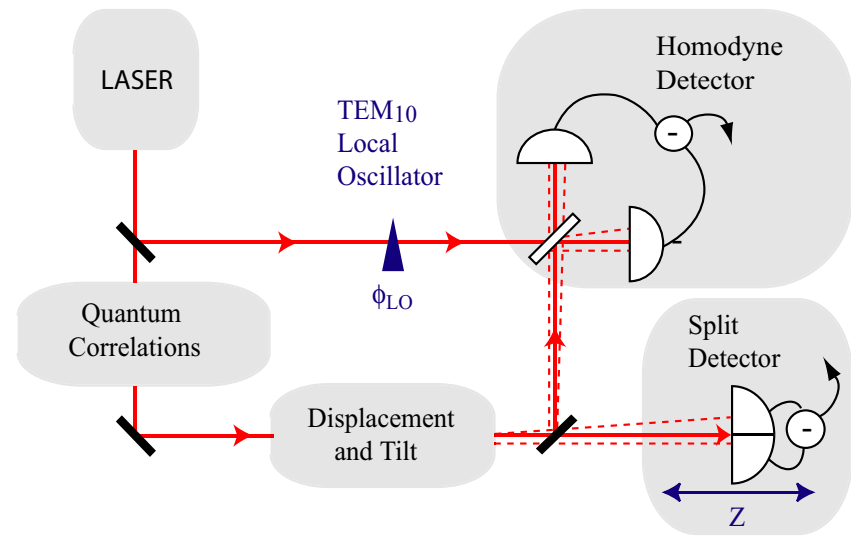

FIG. 1. (Color online) Block diagram of our experimental setup. We compare the performances of two measurement schemes-split detection and homodyne detection with a $\mathrm{TEM}_{10}$ local oscillator-to retrieve displacement and tilt of a Gaussian beam. We show how to modify the input beam using quantum correlations to perform measurements beyond the quantum noise limit. 
(a) Displaced beam

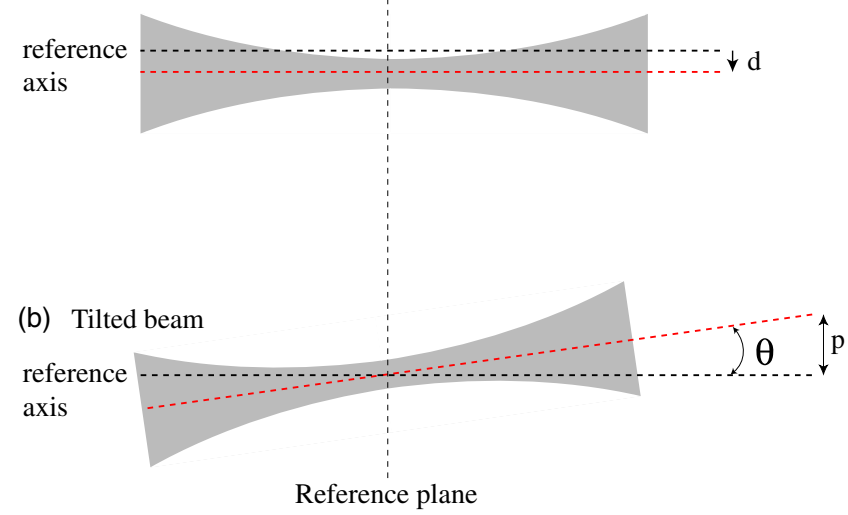

FIG. 2. (Color online) Displacement and tilt definition. In the two-dimensional case, the displacement $d$ corresponds to a translation of the beam in the transverse direction. The tilt $\theta$ corresponds to a angular displacement of the propagation axis.

direction, whereas a tilt corresponds to an angular displacement of the propagation axis by an angle $\theta$. The tilt of a laser beam is linked to the transverse momentum of the beam, in the limit of small angles, given by the following expression:

$$
p=\frac{2 \pi \sin \theta}{\lambda} \simeq \frac{2 \pi \theta}{\lambda},
$$

where $\lambda$ is the optical wavelength. Note that displacement and tilt are defined relative to a particular transverse reference plane. For instance, in Fig. (2), we have chosen the beam waist plane as reference transverse plane.

In the case of small displacement and tilt, i.e., for $d$ $\ll w_{0}$ and $\theta \ll \lambda / w_{0}$-where $w_{0}$ is the beam waist of the incident $\mathrm{TEM}_{00}$ mode, we can Taylor expand the displaced $E_{d}(x)$ and tilted $E_{p}(x)$ Gaussian field to first order, yielding $[1,6]$

$$
\begin{aligned}
& E_{d}(x) \approx E(x)+d \frac{\partial E(x)}{\partial x}, \\
& E_{p}(x) \approx E(x)+i p x E(x) .
\end{aligned}
$$

The equations can be rewritten into

$$
E_{d, p}(x)=A_{0}\left[u_{0}(x)+\left(\frac{d}{w_{0}}+i \frac{w_{0} p}{2}\right) u_{1}(x)\right],
$$

where $u_{n}(x)$ refers to the Hermite Gauss TEM $_{n 0}$ mode [7]. $A_{0}$ is the amplitude of the incident TEM $_{00}$ mode and identifies with the one of the displaced and tilted beam at first order in $d$ and $p$. Equation (4) shows that the information of displacement and tilt of a $\mathrm{TEM}_{00}$ laser beam can be extracted by measuring the $\mathrm{TEM}_{10}$ mode component of the field. Any displacement modulation is transferred to the in-phase amplitude of the TEM $_{10}$ mode relative to the "carrier" $\left(\mathrm{TEM}_{00}\right.$ mode), whereas any tilt modulation is transferred to the $\mathrm{TEM}_{10}$ component in quadrature relative to the $\mathrm{TEM}_{00}$ mode. Note that all transverse modes defined here need a reference frame, which is provided by the axis of the detection device.
Displacement and tilt are thus measured relative to this reference.

In order to give a quantum mechanical description of displacement and tilt of a laser beam, we need to take into account the quantum noise of all the optical modes of the beam, including the vacuum modes. We can write the positive frequency part of the electric field operator in terms of photon annihilation operators $\hat{a}$. The field operator is then given in its more general form by

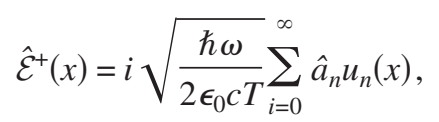

where $\omega$ is the field frequency, $T$ is the integration time, $u_{n}(x)$ are the transverse beam amplitude functions of the $\mathrm{TEM}_{n 0}$ modes, and $\hat{a}_{n}$ are the corresponding annihilation operators. $\hat{a}_{n}$ can be written in the linearization approximation in the form of $\hat{a}_{n}=\left\langle\hat{a}_{n}\right\rangle+\delta \hat{a}_{n}$, where $\left\langle\hat{a}_{n}\right\rangle$ describes the coherent amplitude and $\delta \hat{a}_{n}$ corresponds to the small quantum fluctuations.

We write now the quantum counterpart of Eq. (4), still in the small displacement and tilt regime, in the transverse basis for which $u_{0}$ is the mean field $\mathrm{TEM}_{00}$ mode and $u_{1}$ is the $\mathrm{TEM}_{10}$ mode, and all the other modes are filled with vacuum:

$$
\begin{aligned}
\hat{\mathcal{E}}^{+}(x)= & i \sqrt{\frac{\hbar \omega}{2 \epsilon_{0} c T}}\left\{\sqrt{N}\left[u_{0}(x)+\left(\frac{d}{w_{0}}+i \frac{w_{0} p}{2}\right) u_{1}(x)\right]\right. \\
& \left.+\sum_{i=0}^{\infty} \delta \hat{a}_{n} u_{n}(x)\right\},
\end{aligned}
$$

where $\left|\left\langle\hat{a}_{0}\right\rangle\right|^{2}=N$ is the mean photon number in the entire beam at first order, and where we have introduced the mean value of position and momentum quantum operators of a laser beam, $d=\langle\hat{x}\rangle$ and $p=\langle\hat{p}\rangle$, respectively. These quantum operators are given by

$$
\begin{gathered}
\hat{x}=\frac{w_{0}}{2 \sqrt{N}} \hat{X}_{a_{1}}^{+}, \\
\hat{p}=\frac{i}{w_{0} \sqrt{N}} \hat{X}_{a_{1}}^{-},
\end{gathered}
$$

where we see that position and momentum are linked to the amplitude and phase quadrature of the $\mathrm{TEM}_{10}$ mode component of the field, respectively, given by

$$
\begin{aligned}
& \hat{X}_{a_{1}}^{+}=\left(\hat{a}_{1}+\hat{a}_{1}^{\dagger}\right), \\
& \hat{X}_{a_{1}}^{-}=i\left(\hat{a}_{1}-\hat{a}_{1}^{\dagger}\right) .
\end{aligned}
$$

Moreover, position and momentum are conjugate observables and satisfy the following commutation relation [6]:

$$
[\hat{x}, \hat{p}]=\frac{i}{N} .
$$

In this section, we have defined displacement and tilt of a Gaussian beam, given the general quantum description of 


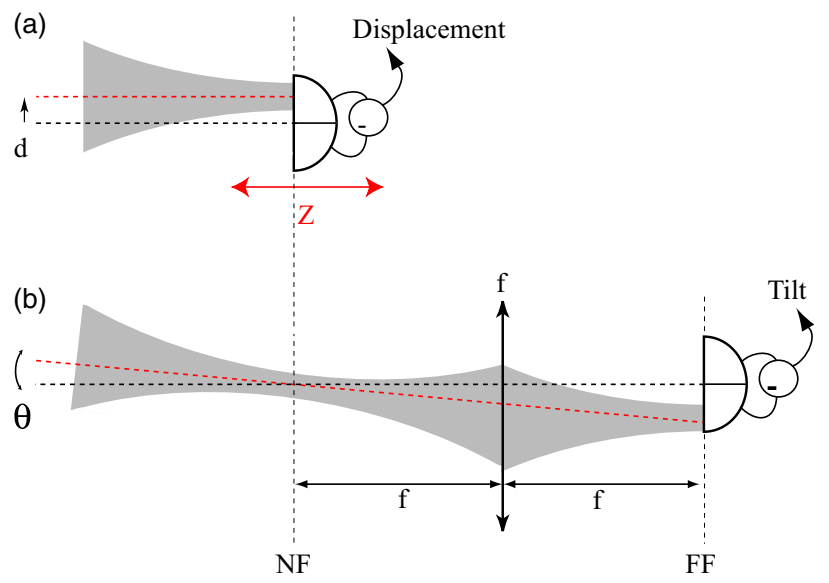

FIG. 3. (Color online) Measuring displacement and tilt of a Gaussian beam with a split detector. Taking a reference plane where displacement and tilt components are needed, displacement can be measured directly with a split detection in the near field (NF) of the reference plane, whereas tilt can be accessed in its far field (FF).

such a field, and show that these former quantities were closely linked to position and momentum, two quantum observables. Using this interesting property, Ref. [6] already proposed a scheme for continuous variable spatial entanglement for bright optical beams, involving two beams, respectively, squeezed in position and momentum mixed on a 50:50 beam-splitter.

\section{QUANTUM NOISE LIMIT FOR DISPLACEMENT AND TILT MEASUREMENTS}

The use of classical resources (i.e., coherent laser beams) sets a lower bound to detection performances, which is called the quantum noise limit (QNL) and arises from the random time arrival of photons on the detector. In the case of displacement measurement of a laser beam, the transverse displacement $d_{\mathrm{QNL}}$ of a TEM 00 laser beam corresponding to a signal to noise ratio (SNR) of 1 , is found to be $[8,9]$

$$
d_{\mathrm{QNL}}=\frac{w_{0}}{2 \sqrt{N}},
$$

where $w_{0}$ is the waist of the beam, and $N$ its total number of photons detected in the interval $T=1 / \mathcal{B}_{\text {res }}$, where $\mathcal{B}_{\text {res }}$ is the resolution bandwidth. Ideally, $T$ is maximized according to the stability of the physical system. For instance in the case of bits read-out in optical disc devices, $\mathcal{B}_{\text {res }}$ roughly corresponds to the scanning frequency. For a $100 \mu \mathrm{m}$ waist, $1 \mathrm{~mW}$ of power at a wavelength of $\lambda=1 \mu \mathrm{m}$, with $\mathcal{B}_{\text {res }}$ $=100 \mathrm{kHz}$, the quantum noise limit is for instance given by $d_{\mathrm{QNL}}=0.2 \mathrm{~nm}$. Note that during test or characterization procedures, the precision can be increased by averaging with the spectrum analyzer, for instance by reducing the video bandwidth $\left(\mathcal{B}_{\text {vid }}\right)$. The QNL effectively corresponds to the minimum measurable displacement when $\mathcal{B}_{\text {vid }}=\mathcal{B}_{\text {res }}$, without averaging.

Similarly, the QNL for momentum measurements can be defined as [6]

$$
p_{\mathrm{QNL}}=\frac{1}{w_{0} \sqrt{N}} .
$$

In the same conditions as the ones defined above, the QNL for momentum measurement is $p_{\mathrm{QNL}}=4 \times 10^{-2} \mathrm{~m}^{-1}$, corresponding to a tilt angle of $\theta_{\mathrm{QNL}}=7 \mathrm{nrad}$.

In order to perform measurements beyond the QNL, i.e., for a given $T$, we have shown in Ref. [10] that filling the appropriate transverse mode of the input field with squeezed light is a necessary and sufficient condition. We call this mode the noise-mode of detection [11].

For example, using $3 \mathrm{~dB}$ of squeezing in the appropriate component of the beam for a displacement measurement leads to a noise reduction of a factor 2 . The SNR is quadratic in $d$ as the signal corresponds to the intensity of the $\mathrm{TEM}_{10}$ component of the displaced field, and the new quantum limit is thus given by $d_{\mathrm{SQZ}}=d_{\mathrm{QNL}} / \sqrt{2}$. It is important to note that, as imposed by Heisenberg inequalities, the measurement of the conjugated observable - the momentum in this case-is degraded.

\section{SPLIT DETECTION}

\section{A. Theory}

The conventional way to measure the displacement of a laser beam is to use a split detector. As shown in Fig. 3(a), the difference between the intensity on each side of the split detector yields a photocurrent proportional to the displacement. This technique is widely used notably for beam alignments, particle tracking and atomic force microscopy. Nevertheless, such a detection device only accesses the beam position in the detector plane, and is totally insensitive to the orientation of the propagation axis of the beam (i.e., tilt). Consider the evolution of the field operator of Eq. (6) under propagation along the $z$ axis, we get

$$
\begin{aligned}
\hat{\mathcal{E}}^{+}(x, z)= & i \sqrt{\frac{\hbar \omega}{2 \epsilon_{0} c T}}\left[\sqrt{N} u_{0}(x, z)+\sqrt{N}\left(\frac{d}{w_{0}}+i \frac{w_{0} p}{2}\right)\right. \\
& \left.\times u_{1}(x, z) e^{i \phi_{G}(z)}+\sum_{i=0}^{\infty} \delta \hat{a}_{n} u_{n}(x, z) e^{i n \phi_{G}(z)}\right],
\end{aligned}
$$

where $u_{n}(x, z)$ is the Hermite Gauss $\operatorname{TEM}_{n 0}$ mode, $\phi_{G}(z)$ is the Gouy phase shift, such as $\phi_{G}(z)=\arctan \left(z / z_{R}\right)$, where $z_{R}$ is the Rayleigh range of the beam. The amount of displacement and tilt in the split-detector plane varies according to the position of the detection device along the axis $z$, because of the Gouy phase shift (i.e., diffraction). Note that the displacement ratio is exactly inverted between near field, for which $\phi_{G}(0)=0$, and far field, for which $\phi_{G}(\infty)=\pi / 2$. This Fourier transform relation is a well-known result in classical optics, for which a displacement in the focal plane of a simple lens is changed into an angular displacement relative to the propagation axis. Therefore, if the exact amount of tilt and displacement is needed in a particular transverse plane, for instance at $z=0$, displacement can be measured in this plane (or in its near field), whereas tilt can only be accessed in its far field, as presented in Fig. 3(b). 
The field presented in Eq. (14) is detected via a split detector whose position is varied along the $z$ axis. The photocurrent is directly proportional to the difference of intensity incident on the two halves of the detector,

$$
\hat{I}_{-}(z)=\int_{0}^{\infty} \hat{\mathcal{E}}^{+}(x, z) \hat{\mathcal{E}}^{+\dagger}(x, z) d x-\int_{-\infty}^{0} \hat{\mathcal{E}}^{+}(x, z) \hat{\mathcal{E}}^{+\dagger}(x, z) d x,
$$

where the center of the split detector itself is chosen as the reference frame, i.e., as the origin of the transverse axis $x$. The beam displacement is thus measured relative to the position of the split detector. Any perturbation modifying this position will create artificial beam displacement. Consequently, we have performed our measurements at an RF frequency ( $4 \mathrm{MHz}$ as presented later on), far from the usual mechanical or thermal instabilities in optical setups, that would potentially generate classical noise on the detector position.

Replacing $\hat{\mathcal{E}}^{+}(x, z)$ with the previous expression yields, for very small displacement and tilt

$$
\begin{aligned}
\hat{I}_{-}(z)= & \frac{\hbar \omega}{2 \epsilon_{0} c T}\left[2 N c_{1}\left(\frac{d}{w_{0}} \cos \phi_{G}(z)+\frac{w_{0} p}{2} \sin \phi_{G}(z)\right)\right. \\
& \left.+\sqrt{N} \sum_{p=0}^{\infty} c_{2 p+1} \delta \hat{X}_{2 p+1}^{-(2 p+1) \phi_{G}(z)}\right]
\end{aligned}
$$

where $\delta \hat{X}_{n}^{\phi}=\delta \hat{a}_{n} e^{-i \phi}+\delta \hat{a}_{n}^{\dagger} e^{i \phi}$ refers to the noise of the quadrature of the $\mathrm{TEM}_{n, 0}$ mode defined by the angle $\phi$, and

$$
c_{n}=\int_{0}^{\infty}-\int_{-\infty}^{0} u_{n}(x) u_{0}(x) d x=\int_{-\infty}^{\infty} u_{n}(x) u_{f}(x) d x,
$$

where $u_{f}$ is the flipped mode, which is a TEM $\mathrm{T}_{00}$ mode whose transverse profile has a $\pi$ phase shift at the origin for $z=0$ [13]. Its decomposition in the $\mathrm{TEM}_{p q}$ basis during propagation is given by

$$
u_{f}(x, z)=\sum_{p=0}^{\infty} c_{2 p+1} u_{2 p+1}(x, z) e^{i(2 p+1) \phi_{G}(z)}
$$

and the fluctuations of its amplitude quadrature operator are found to be

$$
\delta \hat{X}_{f}^{+}=\sum_{p=0}^{\infty} c_{2 p+1} \delta \hat{X}_{2 p+1}^{+},
$$

where $\delta \hat{X}_{2 p+1}^{+}$corresponds to the fluctuations of the amplitude quadrature of the mode $u_{2 p+1}(x, z)$.

Experimentally, we measure the displacement for different split-detector positions. This displacement is induced by a modulating device generating at $z=0$ displacement and tilt modulations of amplitude $d$ and $p$, respectively. A measurement at the modulation frequency, using a spectrum analyzer yields the modulation signal as well as the noise at this frequency. As usual in quantum optics, all equations are directly transposable into the frequency domain. Using Eq. (16), the variance measured by a spectrum analyzer at the precise modulation frequency is given by

$$
\begin{aligned}
V_{\mathrm{SD}}(z)= & \kappa N T\left(\frac{\hbar \omega}{2 \epsilon_{0} c T}\right)^{2}\left[4 ( N T ) \frac { 2 } { \pi } \left(\frac{d}{w_{0}} \cos \phi_{G}(z)\right.\right. \\
& \left.+\frac{p w_{0}}{2} \sin \phi_{G}(z)\right)^{2} \\
& \left.+\left\langle\left(\sum_{p=0}^{\infty} c_{2 p+1} \delta \hat{X}_{2 p+1}^{-(2 p+1) \phi_{G}(z)}\right)^{2}\right\rangle\right],
\end{aligned}
$$

where $\kappa$ is a constant depending only on the electronic gains of the spectrum analyzer, $T=1 / \mathcal{B}_{\text {res }}$ is the integration time and $c_{1}=\sqrt{2 / \pi}$. The first and second bracketed term in Eq. (20), respectively, correspond to modulation signal and noise. In the plane of the modulating device (i.e., for $z=0$ ), the noise term can be written $\left\langle\delta \hat{X}_{f}^{+}\right\rangle$and corresponds to the noise of the amplitude quadrature of the flipped mode. The flipped mode is therefore the only mode contributing to the noise in this particular plane. Note that this is not the case all along the propagation axis. For a coherent incoming beam, this noise term defines the shot noise level, and is equal to 1 . Note that using nonclassical resources for which $\left\langle\delta \hat{X}_{f}^{+2}\right\rangle<1$ in the detection plane results in noise reduction. This case will be discussed in Sec. VI.

The SNR for a coherent beam is found from Eq. (20),

$$
\mathrm{SNR}_{\mathrm{SD}}=4 N T \frac{2}{\pi}\left(\frac{d}{w_{0}} \cos \phi_{G}(z)+\frac{p w_{0}}{2} \sin \phi_{G}(z)\right)^{2} .
$$

As stated in Sec. III, the SNR has a quadratic dependence in displacement $d$ and momentum $p$.

\section{B. Experiment}

We have performed split-detection measurements of displacement and tilt of a Gaussian beam, by moving the position of the detector along the propagation axis, as shown in Fig. 4. Displacement and tilt are produced by a piezoelectric element (PZT) modulated at $4 \mathrm{MHz}$. A modulation signal at such a high frequency has two main advantages. The first one is to operate far from mechanical instabilities, as stated previously. The second one is to be insensitive to the technical noise of our laser source, which is shot noise limited above $1 \mathrm{MHz}$. Each measurement along the propagation axis refers to a different quadrature of the modulation (i.e., a different mixture of displacement and tilt modulation). The results are normalized to the shot noise and taken with a $4.2 \mathrm{~mW}$ incident beam, ensuring $7 \mathrm{~dB}$ of clearance between the shot noise and the electronic noise level. From these measurements, we can infer the displacement and tilt relative amplitude modulation in the PZT plane where the waist is $106 \mu \mathrm{m}$. The displacement signal, accessible in the near field of the PZT, is found to be much smaller than the tilt signal, and even so that it cannot clearly be distinguished from the shot noise. This unusual behavior of the piezoelectric material arises from the operation regime, where the modulation 


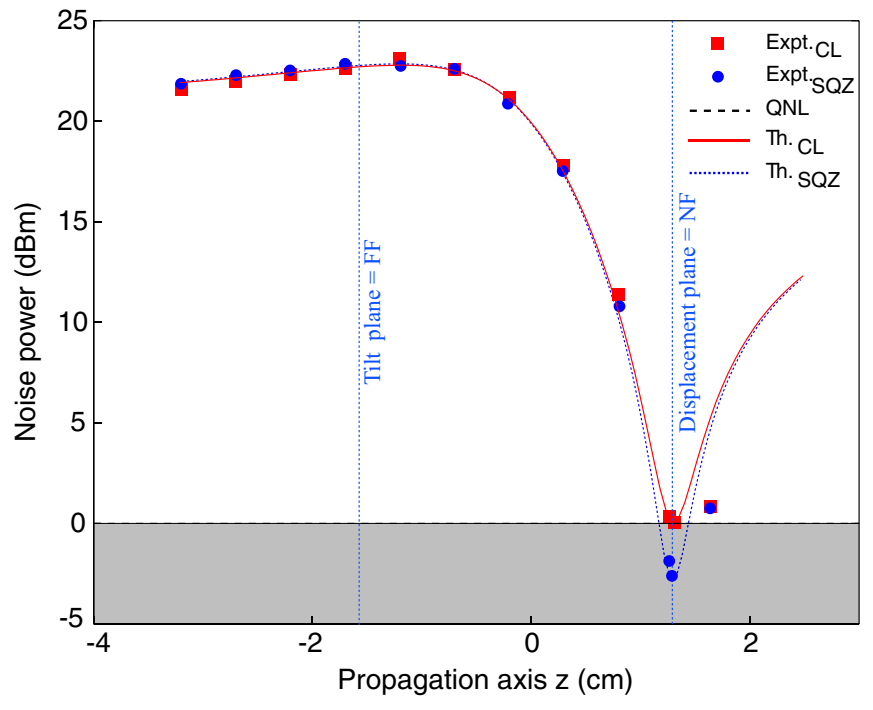

FIG. 4. (Color online) Modulation measurement normalized to the shot noise along the propagation axis of a tilted and displaced beam, using a split detector. The modulation was produced by a PZT at $4 \mathrm{MHz}$, the near field image is located $1.6 \mathrm{~cm}$ after the waist plane which is taken as the reference position $z=0$. The modulation detected in this plane (NF) corresponds to the displacement modulation and represents only $10 \%$ of the overall modulation strength. The tilt information lies in its far field (FF). Classical and nonclassical experimental and theoretical results are presented.

is generated via an accidental mechanical resonance of the PZT. The theoretical curve has been plotted for a coherent illumination, using Eq. (20) for 10\% displacement modulation, and $90 \%$ tilt modulation, ratio determined with the more accurate results presented in Sec. IV. There is a very good agreement with the experimental data. The last experimental point in Fig. 4 lies below the theoretical prediction, as the beam started to be apertured by the split detector, leading to a smaller measured modulation. Note that for technical reasons, our experimental setup is slightly different from the simplest setup presented in Fig. 3, where the reference plane coincides with the beam waist position. As shown on Fig. 4, the waist position lies at $1.6 \mathrm{~cm}$ for the near field of the PZT in our imaging setup.

We have shown in this section how to retrieve displacement and tilt information from a Gaussian beam with a split detector, and have taken experimental results which will be used as a reference in the following sections.

\section{HOMODYNE DETECTION WITH A TEM 10 LOCAL OSCILLATOR}

We have proved theoretically in Ref. [1] that split detection was nonoptimal to retrieve displacement information, as it is only sensitive to the flipped mode instead of the $\mathrm{TEM}_{10}$ mode component of the input field. In order to extract all the displacement and tilt information with up to $100 \%$ efficiency, we propose a homodyne detector involving a $\mathrm{TEM}_{10}$ mode local oscillator, which selects the $\mathrm{TEM}_{10}$ mode component of the field.

In the homodyne detection scheme, two beams are mixed on a 50:50 beam-splitter. The first one is the signal beam containing the displacement and tilt modulations, whose field operator is given by Eq. (6). The second one is the local oscillator (LO), whose field operator is

$$
\hat{\mathcal{E}}_{\mathrm{LO}}^{+}(x)=i \sqrt{\frac{\hbar \omega}{2 \epsilon_{0} c T}}\left(\sqrt{N_{\mathrm{LO}}} u_{1}(x)+\sum_{i=0}^{\infty} \delta \hat{a}_{\mathrm{LO}_{n}} u_{n}(x)\right),
$$

where $N_{\mathrm{LO}}$ denotes the number of photons per second in the beam, and $\phi_{\mathrm{LO}}$ is the local oscillator phase. This definition of the LO profile, namely its axis and spatial extension, sets a spatial reference for beam displacement and tilt measurements, similarly to the position of the split detector in the preceding section. Since displacement and tilt modulations are very small and the local oscillator is much brighter than the signal beam (i.e., $N_{\mathrm{LO}} \gg N$ ), the usual calculation of the intensity difference between the two homodyne detectors at the modulation frequency gives

$$
\begin{aligned}
\hat{I}_{-}= & \frac{\hbar \omega}{2 \epsilon_{0} c T}\left[2 \sqrt{N N_{\mathrm{LO}}}\left(\frac{d}{w_{0}} \cos \phi_{\mathrm{LO}}+\frac{w_{0} p}{2} \sin \phi_{\mathrm{LO}}\right)\right. \\
& \left.+\sqrt{N_{\mathrm{LO}}} \delta \hat{X}_{1}^{\phi_{\mathrm{LO}}}\right],
\end{aligned}
$$

and similarly to Eq. (20), its variance at the displacement and tilt modulation frequency is therefore

$$
\begin{aligned}
V_{\mathrm{HD}}\left(\phi_{\mathrm{LO}}\right)= & \kappa N_{\mathrm{LO}} T\left(\frac{\hbar \omega}{2 \epsilon_{0} c T}\right)^{2}\left[4 N T \left(\cos \left(\phi_{\mathrm{LO}}\right) \frac{d}{w_{0}}\right.\right. \\
& \left.+\sin \left(\phi_{\mathrm{LO}}\right) \frac{p w_{0}}{2}\right)^{2}+\left\langle\delta \hat{X}_{1}^{\phi_{\mathrm{LO}}^{2}}\right]
\end{aligned}
$$

where the constant $\kappa$ is identical to the split-detection part as long as the spectrum analyzer settings have not been changed. The first bracketed term corresponds to the modulation signal. The second one refers to the noise of the $\mathrm{TEM}_{10}$ component of the detected field, and its variation with the local oscillator phase $\phi_{\mathrm{LO}}$ is given by $\left\langle\delta \hat{X}_{1}^{\phi_{\mathrm{LO}}^{2}}\right\rangle$ $=\left\langle\delta \hat{X}_{1}^{+2}\right\rangle \cos ^{2} \phi_{\mathrm{LO}}+\left\langle\delta \hat{X}_{1}^{-2}\right\rangle \sin ^{2} \phi_{\mathrm{LO}}$, where $\left\langle\delta \hat{X}_{1}^{+{ }^{2}}\right\rangle$ and $\left\langle\delta \hat{X}_{1}^{-2}\right\rangle$ are the noise of the amplitude and phase quadrature of the $\mathrm{TEM}_{10}$ mode, respectively. Scanning the local oscillator phase allows to measure all the quadratures of the displacement and tilt modulation. We have omitted the Gouy phase shift in the previous expression, as it can be incorporated as a constant term in the local oscillator phase. This phase is still defined so that $\phi_{\mathrm{LO}}=0$ corresponds to a displacement measurement in the PZT plane.

Only the $\mathrm{TEM}_{10}$ mode of the incoming beam contributes to the noise, as it matches the local oscillator transverse shape. All the other modes contributions cancel out since they are orthogonal to the local oscillator. The TEM $_{10}$ mode is thus the noise mode of the homodyne detection [11], and precisely matches the information to be extracted. We can show, using a Cramer Rao bound estimation, that the $\mathrm{TEM}_{10}$ homodyne detection is an optimal displacement and tilt detection, as no other device can possibly perform such measurements with a better SNR [12]. 
For a coherent incoming $\mathrm{TEM}_{10}$ mode, the previous noise term defines the shot noise level, and is equal to 1 . Using squeezed light in the $\mathrm{TEM}_{10}$ mode component of the incoming beam would result in noise reduction, and will be discussed in Sec. VI.

The SNR for a coherent incoming beam can be derived from Eq. (22) in the homodyne detection case

$$
\mathrm{SNR}_{\mathrm{HD}}=4 N T\left(\frac{d}{w_{0}} \cos \phi_{\mathrm{LO}}+\frac{p w_{0}}{2} \sin \phi_{\mathrm{LO}}\right)^{2} .
$$

Comparing the split and homodyne detections schemes yields certain similarities between Eqs. (20) and (22). First, a variation of the local oscillator phase $\phi_{\mathrm{LO}}$ in the homodyne scheme is equivalent to a propagation along the $z$ axis inducing a Gouy phase shift $\phi_{G}$ in the split detection case. Second, an additional $2 / \pi$ geometry factor in the split-detection case arises from the imperfect overlap between the flipped mode and the $\mathrm{TEM}_{10}$ mode, as discussed in Ref. [1]. The comparison between the two SNRs in the coherent case yields a theoretical efficiency ratio given by

$$
R_{\mathrm{th}}=\frac{\mathrm{SNR}_{\mathrm{SD}}}{\mathrm{SNR}_{\mathrm{HD}}}=\frac{2}{\pi} \frac{N_{\mathrm{SD}}}{N_{\mathrm{HD}}},
$$

where $N_{\mathrm{SD}}$ and $N_{\mathrm{HD}}$ refer to the number of photons per second in the displaced and tilted beam, for the split-detection and the homodyne-detection case, respectively. For identical signal beams powers, this means that the split detection is only $2 / \pi=64 \%$ efficient compared to the homodyne detection. Using the homodyne detection thus corresponds to an improvement of $(100-64) / 64=56 \%$. Note that this power efficiency corresponds to the $80 \%$ amplitude efficiency of the split-detection stated in Refs. $[1,11]$

Eventually, the intensity factor before the bracketed term in Eq. (22) and Eq. (20) can be much bigger in the homodyne-detection case, as it corresponds to the local oscillator intensity instead of the input beam intensity in the split-detection case. It is thus easier to have more electronic noise clearance in the homodyne case for weak signals.

In this section, we have shown-still theoretically-how to retrieve displacement and tilt using a homodyne detector with a TEM $_{10}$ local oscillator. Moreover, we have proved a $56 \%$ theoretical improvement of this scheme compared to the split detection.

\section{DISPLACEMENT AND TILT MEASUREMENTS BEYOND THE QUANTUM NOISE LIMIT}

When the information to be retrieved is below-or of the order of - the quantum noise, nonclassical resources (i.e., squeezed laser beams) can help extract the information. For each type of detection (i.e., homodyne and split detection), the only transverse mode component within the incident field which contributes to the noise has been identified in the preceding sections. The noise modes of the split and homodyne detection are the flipped mode and the $\mathrm{TEM}_{10}$ mode, respectively. Since displacement and tilt of a $\mathrm{TEM}_{00}$ beam lies in
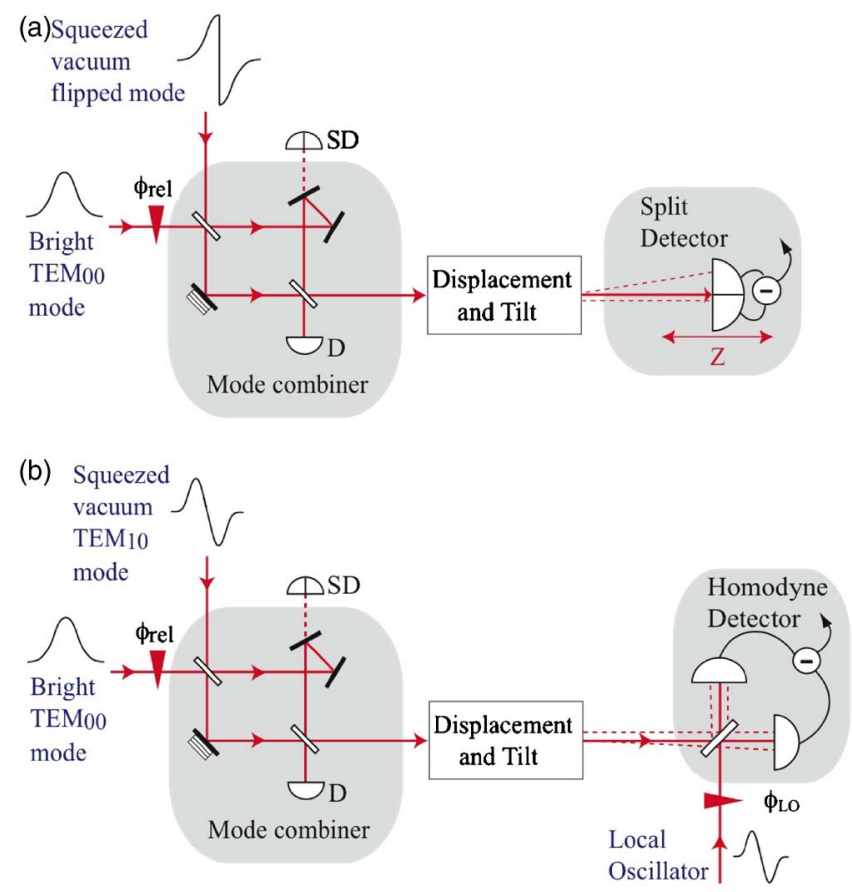

FIG. 5. (Color online) Schematic of displacement and tilt modulation measurement beyond the quantum noise limit. (a) With a split detector and (b) with a homodyne detector. Prior to the modulation generated via a PZT at a few MHz, a bright TEM $_{00}$ beam is combined without losses with a squeezed vacuum noise mode. This was done with a special Mach-Zehnder which has an additional mirror in one arm. A mirror leakage is used to lock the relative phase between both input modes. All different combinations of displacement and tilt modulations are accessible when (a) the position of the split detector along the propagation axis $z$ is varied, and (b) when the local oscillator phase $\phi_{\mathrm{LO}}$ is scanned.

the TEM $_{10}$ component of the beam, noise mode and information encoded are matched for the homodyne detection only, accounting for the nonoptimal split detection.

Subshot noise measurements with both schemes can be performed using the setups shown in Fig. 5, by filling the noise mode of the input beam with squeezed light. A mode combiner must be used to merge the signal beam-in our case a bright $\mathrm{TEM}_{00}$ beam-with the noise mode of detection, filled with squeezed vacuum. Note that it must be a vacuum mode, or a very dim field not to contribute to the signal, but only to reduce the quantum noise properties. The combination of beams cannot be done with a sheer beamsplitter as the squeezing is not robust to losses. Instead, we used a special Mach-Zehnder interferometer with an additional mirror in one arm, see (Fig. 5). This mirror has no effect on even transverse profiles, but induces an additional $\pi$ phase shift to odd transverse profiles. Therefore, thanks to this asymmetry, orthogonal even and odd modes, which are incident on the two input ports of the Mach-Zehnder, interfere constructively on the same output port without experiencing any losses. The integrality of the bright beam and the squeezing of the squeezed vacuum mode-(a) flipped mode or (b) $\mathrm{TEM}_{10}$ mode-are thus preserved at the output of the interferometer. Note that other devices can be used $[9,14,15]$.

In order to make a direct comparison of the performances of the split detection and the homodyne detection, we have 


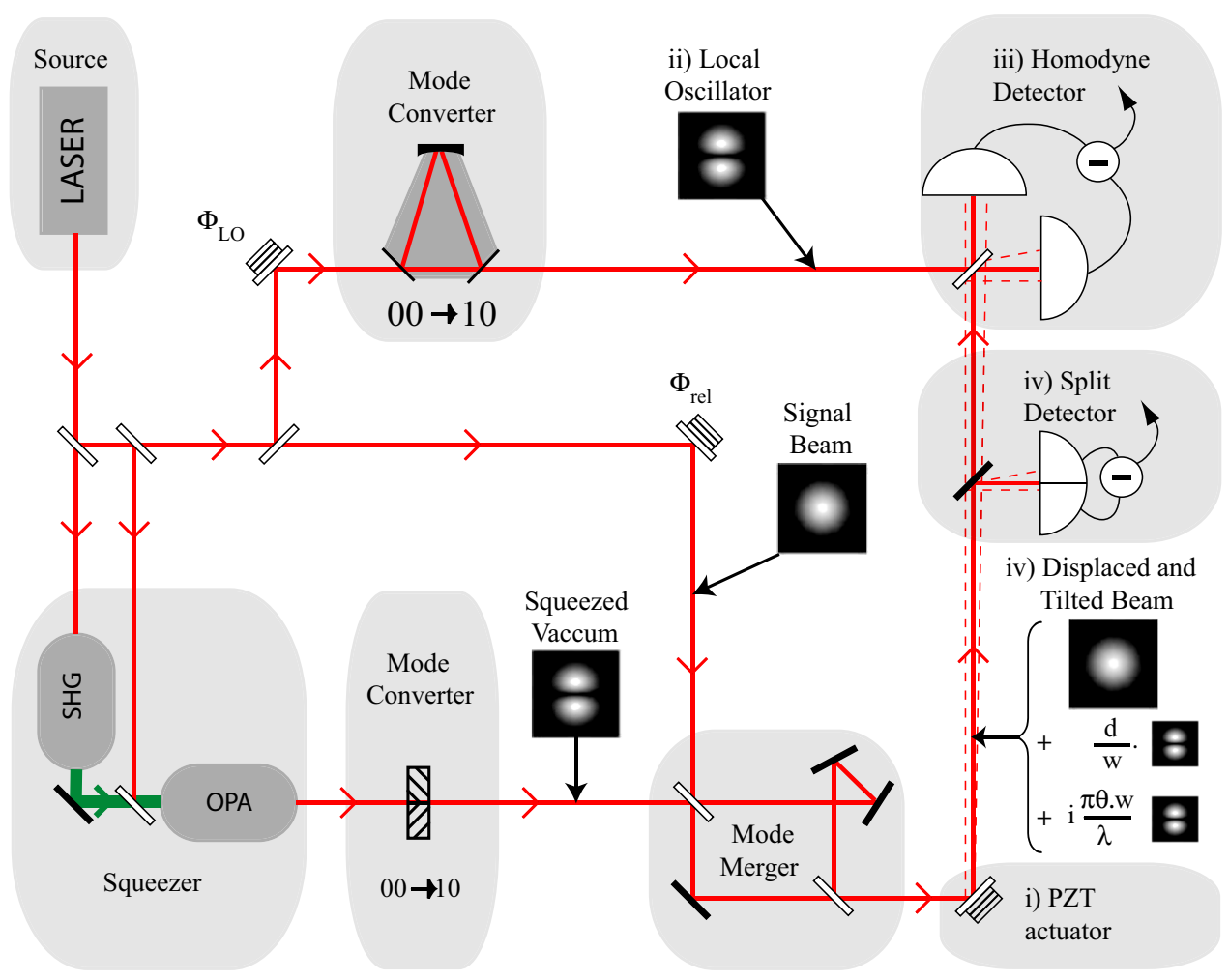

FIG. 6. (Color online) Experimental scheme to measure displacement and tilt with a split detector and a homodyne detector with the same operating conditions.

built the experimental setup sketched in Fig. 6, where both schemes are tested in the same operating conditions. In addition to a simple comparison involving only classical resources, we designed the experience in order to allow measurements beyond the QNL. At this stage, we were unable to produce directly a squeezed $\mathrm{TEM}_{10}$ mode, we have therefore chosen to generate a squeezed flipped mode, which also corresponds to a squeezed $\mathrm{TEM}_{10}$ mode having experienced $36 \%$ losses. Indeed, the amount of squeezing in the amplitude quadrature of the $\mathrm{TEM}_{10}$ component of the flipped mode can be deduced from

$$
\left\langle\delta \hat{X}_{1}^{+2}\right\rangle=\frac{2}{\pi}\left\langle\delta \hat{X}_{f}^{{ }^{2}}\right\rangle+\left(1-\frac{2}{\pi}\right),
$$

as all the modes except the flipped mode are filled with coherent light. If the flipped mode is a classical coherent beam, $\left\langle\delta \hat{X}_{f}^{+2}\right\rangle=1$, which also implies that the $\mathrm{TEM}_{10}$ component is coherent $\left\langle\delta \hat{X}_{1}^{+}{ }^{2}\right\rangle=1$, as expected. In the end, if we start with $3.6 \mathrm{~dB}$ of squeezing in the amplitude quadrature of the flipped mode as discussed below, we get $2 \mathrm{~dB}$ of squeezing in the $\mathrm{TEM}_{10}$ mode, which is exactly what can be measured experimentally by using the homodyne detection with a $\mathrm{TEM}_{10}$ local oscillator.

We used the following experimental procedure. First we generated a $3.6 \mathrm{~dB}$ squeezed $\mathrm{TEM}_{00}$ mode from a monolithic optical parametric amplifier (OPA) pumped by a frequency doubled YAG laser delivering $600 \mathrm{~mW}$ at $1064 \mathrm{~nm}$, and seeded by a $\mathrm{TEM}_{00}$ mode. This very low power (nW) squeezed beam then experiences a mode conversion into the flipped mode thanks to a special wave-plate made of two half-wave plates whose optical axis have been rotated $90^{\circ}$ relative to each other [13]. A beam incident on such an optical element yields a $\pi$ phase shift on one-half of its transverse profile.

Thanks to the special Mach-Zehnder interferometer formerly presented, we combine this beam with a bright $\mathrm{TEM}_{00}$ beam, therefore preserving their potential nonclassical properties. To achieve this experimentally, we first mode matched both input beams of the interferometer without the special wave plate, reaching $99.5 \%$ visibility on the first beam splitter of the interferometer. The squeezed beam, although very dim, is still bright enough to be mode matched with the other bright $\mathrm{TEM}_{00}$ beam. The interferometer is then aligned on the OPA beam without the wave plate with $98 \%$ visibility and then the wave plate is slid in the center of the beam to a maximum visibility of $97.8 \%$. Note that we purposely introduced a leakage in one of the mirrors to lock the relative phase $\phi_{\text {rel }}$ between the two input modes with a split detector (SD), as drawn in Fig. 5. In the end, the global mode combiner efficiency is still higher than $97 \%$.

The multimode squeezed beam hereby generated is then displaced and tilted with a PZT, as presented in Sec. IV, and the information is detected with either one of the split or homodyne detection schemes. Let us first briefly concentrate on the results obtained with the split detector, in presence of the squeezed flipped mode. We see in Fig. 4 that we were able to measure a modulation below the QNL. These measurements precisely correspond to detection in the near field of the mode converting wave-plate-which is also the far field of the PZT - as the flipped mode is not stable with 
propagation, and the squeezing degrades very quickly along the $z$ direction. Note that the excess noise expected far from the near field plane cannot be resolved because of the strong detected modulation in this region.

We now focus on the $\mathrm{TEM}_{10}$ homodyne detection. The $\mathrm{TEM}_{10}$ local oscillator is produced with a misaligned ring cavity locked to resonance on the $\mathrm{TEM}_{10}$ mode represented in Fig. 6. The cavity has been designed such that it delivers a pure transverse output mode (i.e., high order modes are not simultaneously resonant in the cavity). We mode matched this local oscillator beam to the signal beam by previously locking the ring cavity to the $\mathrm{TEM}_{00}$ mode resonance, reaching a visibility of $98.9 \%$ with the $\mathrm{TEM}_{00}$ input mode.

The experimental results, obtained with the spectrum analyzer in zero-span mode at $4 \mathrm{MHz}$, are presented in Fig. 7(a) and Fig. 7(b), when the $\mathrm{TEM}_{10}$ local oscillator phase is scanned and locked for displacement $\left(\phi_{\mathrm{LO}}=0\right)$ and tilt $\left(\phi_{\mathrm{LO}}=\pi / 2\right)$ measurement. The electronic noise is $11.7 \mathrm{~dB}$ below the shot noise level. All traces are corrected for this noise. Without the use of squeezed light, the displacement modulation cannot clearly be resolved, as in the split detection case. Improvement of the SNR for displacement measurement beyond the quantum noise limit is achieved when the squeezed quadrature of the $\mathrm{TEM}_{10}$ mode is in phase with the displacement measurement quadrature (i.e., in phase with the incoming $\mathrm{TEM}_{00}$ mode). Since we are dealing with conjugate variables, improving the displacement measurement degrades the tilt measurement of the same beam, as required by the antisqueezing of the other quadrature. The displacement measurement is improved by the $2 \mathrm{~dB}$ of squeezing, whereas the tilt measurement is degraded by the $8 \mathrm{~dB}$ of antisqueezing. Theoretical curves calculated with $2 \mathrm{~dB}$ of noise reduction and $8 \mathrm{~dB}$ of antisqueezing, and $90 \%$ of tilt modulation and $10 \%$ of displacement modulationcontinuous curves in Fig. 7(a) — are in very good agreement with experimental data. In our experiment, we have a TEM 00 waist size of $w_{0}=106 \mu \mathrm{m}$ in the PZT plane, a power of $170 \mu \mathrm{W}, \mathcal{B}_{\text {res }}=100 \mathrm{kHz}$ and $\mathcal{B}_{\text {vid }}=100 \mathrm{~Hz}$, corresponding to a QNL of $d_{\mathrm{QNL}}=0.6 \mathrm{~nm}$. The measured displacement lies $0.5 \mathrm{~dB}$ above the squeezed noise floor, yielding a displacement modulation 0.08 times larger than the QNL. As the modulation has a square dependence on the displacement $d$, we get $d_{\text {expt }}=\sqrt{0.08} d_{\mathrm{QNL}}=0.15 \mathrm{~nm}$. This would correspond to a trace $0.3 \mathrm{~dB}$ above the QNL [trace MOD: $\phi_{\mathrm{LO}}=0$ in Fig. 7(a)]. The use of squeezed light thus allows a more accurate determination of the displacement signal. The ratio between displacement and tilt modulations can be inferred from the theoretical fit in Fig. 7, giving a measured tilt of $0.13 \mu \mathrm{rad}$.

We have in this section demonstrated measurements of a pair of quantum conjugate variables-displacement and tilt-with a homodyne detector involving a $\mathrm{TEM}_{10}$ mode local oscillator, and performed subshot noise displacement measurements.

\section{COMPARISON}

Split-detection and homodyne detection efficiencies would ideally be compared exactly in the same regime.
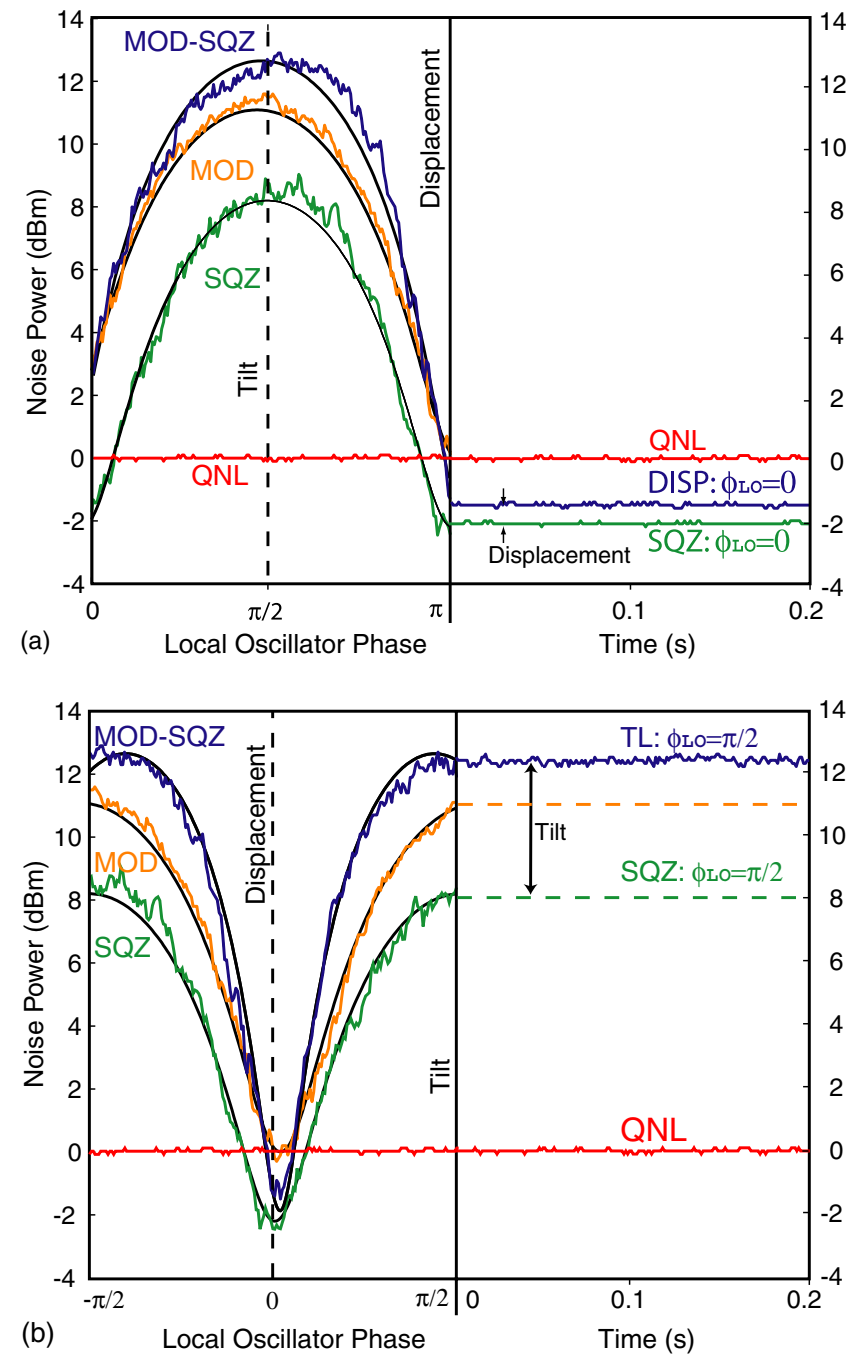

FIG. 7. (Color online) Demonstration of subshot noise measurements of (a) displacement and (b) tilt modulations using spatial homodyne detector. The figures show an example with $90 \%$ of tilt, and $10 \%$ of displacement modulations. Left-hand side of the figures shows the scanning of the local oscillator phase $\phi_{\mathrm{LO}}$ that continuously access the pure displacement (at $\phi_{\mathrm{LO}}=0$ and $\pi$ ) to pure tilt (at $\phi_{\mathrm{LO}}=\pi / 2$ and $3 \pi / 2$ ) information of the beam. QNL, quantum noise limit. SQZ, quadrature noise of squeezed light with $2 \mathrm{~dB}$ of squeezing and $8 \mathrm{~dB}$ of antisqueezing on the $\mathrm{TEM}_{10}$ mode, but without any modulation signal. MOD, measured modulation with coherent light. MOD-SQZ, measured modulation with squeezed light. Right-hand side of the figures shows the corresponding locked local oscillator phase to the (a) displacement or (b) tilt measurement. $\mathrm{SQZ}$, at $\phi_{\mathrm{LO}}=0$ the squeezed noise level is $2 \mathrm{~dB}$ below the shot noise and at $\phi_{\mathrm{LO}}=\pi / 2$ there is $8 \mathrm{~dB}$ of antisqueezing noise. DISP: MOD-SQZ curve locked to $\phi_{\mathrm{LO}}=0$ for displacement measurement. TL, MOD-SQZ curve locked to $\phi_{\mathrm{LO}}=\pi / 2$ for tilt measurement. Displacement measurement is improved by the $2 \mathrm{~dB}$ of squeezing, while the tilt measurement is degraded by the $8 \mathrm{~dB}$ of antisqueezing.

However, the optimum operating regimes for both schemes are not compatible. Indeed, on the one hand, in the splitdetection case, the incident beam power must be "intense" in order to have enough clearance relative to the electronic 
noise level, i.e., several $\mathrm{mW}$ for our split-detection device. On the other hand, in the homodyne-detection case, the local oscillator must to be the "intense" beam, and is the one whose power must ensure an electronic noise clearance. Its power must thus be of the order of the $\mathrm{mW}$ for our homodyne detectors. In this case the power of the incident displaced and tilted beam should beat at least 10 times less than the power of the LO beam, i.e., its power cannot exceed about $100 \mu \mathrm{W}$. In order to provide a comparison, we use Eq. (24) which takes into account the power discrepancy between both experiments. Note however that all other parameters are identical, as shown by the symmetry of the system shown in Fig. 6.

The experimental efficiency ratio can be accessed by the ratio of maximum modulation power relative to the shot noise $\operatorname{Mod}_{\mathrm{SD}}$ and $\mathrm{Mod}_{\mathrm{HD}}$, respectively, detected with the split detection and the homodyne detection,

$$
R_{\text {expt }}=\frac{\operatorname{Mod}_{\mathrm{SD}}}{\operatorname{Mod}_{\mathrm{HD}}} .
$$

The two experimental curves presented in Figs. 4 and 7(a) (MOD) read $\operatorname{Mod}_{\mathrm{SD}}=23 \mathrm{~dB}$ and $\mathrm{Mod}_{\mathrm{HD}}=11.3 \mathrm{~dB}$, for beam powers of $P_{\mathrm{SD}}=4.2 \mathrm{~mW}$ and $P_{\mathrm{HD}}=170 \mu \mathrm{W}$, respectively. Using Eqs. (24) and (26), we can compare our theoretical prediction and the experimental efficiency ratio with the following ratio:

$$
\frac{R_{\text {expt }}}{R_{\text {th }}}=1 \pm 0.05,
$$

where the uncertainty is mainly due to the determination of the maximum modulation values. We therefore report an efficiency improvement of $56 \%$, in perfect agreement with the theoretical value calculated in Ref. [1].

Let us now compare the advantages or drawbacks of both detection devices. Although we have proven that the homodyne scheme is more efficient, it is not always the most convenient or the most appropriate to operate in some experimental setups.

The main advantage of the split detector is obviously its simplicity for a single displacement measurement. When the beam to be analyzed is intense enough to be distinguished from the electronic noise, such a simple device must be preferred. However, as soon as both conjugated variables are investigated, several split detections are required, potentially involving a displacement of the detector itself in between two consecutive measurements. There are additional limitations to the use of a split detector due to its gap and to its finite size, which are imposing constraints when the variation of the modulation on the propagation axis is measured. The accessible range to a good detection on the $z$ axis is small, as the beam can neither be too small (because of the gap), nor too large because of the finite size of the detector.

When it comes to ultrasensitive measurements, the gain in efficiency provided by the homodyne scheme relative to the split detection is a simpler technique than the use of nonclassical light with a split detector. In order to perform measurements beyond the QNL, one must carefully image the squeezed flipped mode onto the sample with which the beam is interacting, and also onto the detector, as the flipped mode is not stable in propagation. As a $56 \%$ improvement roughly corresponds to the use of $3 \mathrm{~dB}$ of squeezing, homodyne detection with coherent illumination should be preferred in this case. Another advantage of the homodyne device is to allow measurements of displacement and tilt of a beam without changing the position of the detector, just by varying the local oscillator phase, which has to be carefully mode matched to the incoming beam. High measurement rates of both variables can thus be achieved. Moreover, weak signals that would have been drowned in the electronic noise of a split detector are measurable as the noise clearance is determined by the intense local oscillator. Nevertheless, using this detection device requires coherent illumination as it relies on interference measurement. Additionally, implementing a local oscillator beam is sometimes impossible, typically when the source of the beam to be analyzed cannot be accessed.

In order to further improve the measurement sensitivity, for instance when the signal is so weak that it cannot be distinguished from the quantum noise, a nonclassical beam with a squeezed $\mathrm{TEM}_{10}$ component can be generated. The homodyne detection noise will be reduced either for displacement or tilt measurement, according to the relative phase between squeezed beam and carrier beam. Such detection can for instance be applied to track the position and orientation-at high frequency not to have thermal and mechanical perturbations - of a phase object or a biological sample-we recall that the use of nonclassical light is limited to transparent propagation media, as the squeezing rapidly decays with losses.

\section{CONCLUSION}

We have demonstrated a homodyne-detection scheme involving a $\mathrm{TEM}_{10}$ mode local oscillator in order to measure the displacement and tilt of a Gaussian beam. We report a detection efficiency improvement of 56\% relative to the split detection, in perfect agreement with the theoretical value. Our detection setup is very simple and could thus easily replace split and quadrant detectors in many applications, particularly when tilt and displacement are needed at the same time. Moreover, further developments using nonclassical are possible, as we have presented measurements beyond the QNL with these devices. Note that we are now able to generate the squeezed $\mathrm{TEM}_{10}$ mode directly, without the use of a wave plate, with a misaligned optical parametric amplifier [16], allowing a simplification of the setup.

Quantum measurements in the transverse plane such as the ones presented in this paper potentially open the way to parallel quantum information processing. Indeed, instead of using amplitude and phase quadratures or Stokes operators, conjugated quantum operators are now available in the transverse plane. The generation of spatial entanglement between position and momentum of two laser beams will be considered as a first step towards this goal. Note that spatial entanglement has already been demonstrated with orbital angular momentum in the single photon regime $[17,18]$. Other types of experiments that could follow are dense coding and teleportation of spatial information and spatial holography. 


\section{ACKNOWLEDGMENTS}

The authors thank Magnus Hsu, Warwick Bowen, and Nicolai Grosse for helpful discussions. This work was sup- ported with funding from the Australian Research Council Centre of Excellence program. Two of the authors (P.K.L. and H-A.B.) acknowledge funding from the ARC.
[1] M. T. L. Hsu, V. Delaubert, P. K. Lam, and W. P. Bowen, J. Opt. B: Quantum Semiclassical Opt. 6, 495 (2004).

[2] E. Morrison, B. J. Meers, D. I. Robertson, and H. Ward, Appl. Opt. 33, 5041 (1994); 33, 5049 (1994).

[3] J. H. Chow, G. de Vine, M. B. Gray, and D. E. McClelland, Opt. Lett. 20, 2339 (2004).

[4] See also references in Ref. [1].

[5] S. M. Barnett and R. Zambrini, J. Mod. Opt. 53, 613 (2006).

[6] M. T. L. Hsu, W. P. Bowen, N. Treps, and P. K. Lam, Phys. Rev. A 72, 013802 (2005).

[7] A. E. Siegman, Lasers (University Science, Mill Valley, CA 1986).

[8] C. Fabre, J. B. Fouet, and A. Maître, Opt. Lett. 25, 76 (2000).

[9] N. Treps, N. Grosse, W. P. Bowen, M. T. L. Hsu, A. Maître, C. Fabre, H-A. Bachor, and P. K. Lam, J. Opt. B: Quantum Semiclassical Opt. 6, S664 (2004).

[10] N. Treps, V. Delaubert, A. Maître, J. M. Courty, and C. Fabre, Phys. Rev. A 71, 013820 (2005).

[11] V. Delaubert, N. Treps, C. C. Harb, P. K. Lam, and H-A. Ba- chor, Opt. Lett. 31, 1537 (2006).

[12] V. Delaubert, N. Treps, P. Réfrégier, H-A. Bachor, and C. Fabre, e-print quant-ph/0609188.

[13] V. Delaubert, D. A. Shaddock, P. K. Lam, B. C. Buchler, H-A. Bachor, and D. E. McClelland, J. Opt. A, Pure Appl. Opt. 4, 393 (2002).

[14] N. Treps, U. Andersen, B. Buchler, P. K. Lam, A. Maître, H-A. Bachor, and C. Fabre, Phys. Rev. Lett. 88, 203601 (2002).

[15] N. Treps, N. Grosse, W. P. Bowen, C. Fabre, H.-A. Bachor, and P. K. Lam, Science 301, 940 (2003).

[16] M. Lassen, V. Delaubert, C. C. Harb, P. K. Lam, N. Treps, and H-A Bachor, J. Eur. Opt. Soc. 1, 06003 (2006).

[17] S. S. R. Oemrawsingh, X. Ma, D. Voigt, A. Aiello, E. R. Eliel, G. W. 't Hooft, and J. P. Woerdman, Phys. Rev. Lett. 95, 240501 (2005).

[18] S. S. R. Oemrawsingh, J. A. de Jong, X. Ma, A. Aiello, E. R. Eliel, G. W. 't Hooft, and J. P. Woerdman, Phys. Rev. A 73, 032339 (2006). 